\title{
Raynaud's phenomenon: new aspects of pathogenesis and the role of nailfold videocapillaroscopy
}

\author{
Anna Kuryliszyn-Moskal, Jacek Kita, Anna Hryniewicz \\ Department of Rehabilitation, Medical University of Bialystok, Bialystok, Poland
}

\begin{abstract}
Raynaud's phenomenon (RP) refers to paroxysmal pallor or cyanosis of the digits of the hands or feet and, infrequently, the tips of the nose or ears (acral parts) owing to cold-induced vasoconstriction of the digital arteries, precapillary arterioles, and cutaneous arteriovenous shunts.

Raynaud's phenomenon reflects an exaggeration of normal central and local vasomotor responses to cold or emotion. Raynaud's phenomenon has been classified as primary or secondary, depending on whether it occurs as an isolated condition or is associated mainly with a connective tissue disease.

Dysregulation of autonomic and sensitive nerve fibers, functional and structural vessel changes, and intravascular alterations can be observed in the pathogenesis of RP.

Nailfold videocapillaroscopy (NVC) is the best non-invasive and repetitive diagnostic technique for detecting morpho-functional changes in the microcirculation. Nailfold videocapillaroscopy is accepted in early diagnosis and monitoring of primary and secondary RP.
\end{abstract}

Key words: Raynaud's phenomenon, microvascular abnormalities, nailfold videocapillaroscopy.

Raynaud's phenomenon (RP) is characterized by digital ischemic attacks, followed by cyanosis and erythema. It is usually bilateral, symmetric, mostly seen in young women and is considered as a primary (classic, idiopathic) form and a benign clinical problem. Raynaud's phenomenon could be a manifestation of connective tissue disease, vasculitis, cryoglobulinemia, drug-induced, etc. (considered as secondary). The vasospastic attack of RP is classically manifested as triphasic color changes.

Raynaud's phenomenon refers to paroxysmal pallor or cyanosis of the digits of the hands or feet, and, infrequently, the tips of the nose or ears (acral parts), nipples and, very rarely, the tongue owing to cold-induced vasoconstriction of the digital arteries, precapillary arterioles, and cutaneous arteriovenous shunts [1-3]. Characteristically, the fingers change color in the sequence white-blue-red [4]. The white phase (ischemia) is due to excessive vasoconstriction and cessation of regional blood flow. This phase is followed by a cyanotic phase (blue deoxygenation), as the residual blood in the finger desaturates. The red phase (reperfusion) is due to hyperemia as the attack subsides and blood flow is restored. Structural changes in the arterial walls are absent except late in the course, when intimal thickening can appear. Raynaud's phenomenon reflects an exaggeration of normal central and local vasomotor responses to cold or emotion. The prevalence in the overall population is approximately $3 \%$ to $5 \%$; the median age of those affected is 14 years [1].

The course of RP is usually benign, but in long-standing cases atrophy of the skin, can appear subcutaneous tissues, and muscles, ulceration and ischemic gangrene are rare [5].

Raynaud's phenomenon has been classified as primary (pRP) or secondary (sRP), depending on whether it occurs as an isolated condition or is associated with an underlying disease, mainly a connective tissue disease (CTD-RP) [6]. Raynaud's phenomenon may also occur

Address for correspondence:

Jacek Kita, Department of Rehabilitation, Medical University of Bialystok, M. Skłodowskiej-Curie 24 A, 15-267 Bialystok, Poland,

e-mail: jacek.kita@umb.edu.pl

Submitted: 16.03.2015; Accepted: 4.05.2015 
in other non-CTD (occupational factors, repetitive trauma, malignancies, clotting disease, neurodegenerative cause, nervous system diseases, endocrine diseases, surgical diseases, iatrogenic - drugs, drug addiction).

In contrast to uncomplicated or pRP, sRP refers to arterial insufficiency of the extremities caused by various conditions, including systemic lupus erythematosus, systemic sclerosis (SSc; scleroderma), atherosclerosis, Takayasu's disease or Bürger disease [2, 5, 7].

Since RP may be the first manifestation of such conditions, all patients who manifest the condition should undergo evaluation to rule out an underlying cause, which will be apparent at presentation or will develop later in approximately $10 \%$ of patients [8]. Features sug gestive of secondary RP include age of onset $>30$ years, more severe episodes associated with skin lesions, and clinical features of connective tissue disease.

The agents that may cause RP are physical (low temperature, vibration) and psychological (stress).

The pathophysiology of RP is still not completely understood. The key factor in the pathogenesis of RP is thought to be dysregulation in the control mechanisms (i.e., neural, endothelial, and intravascular) of the vascular motility, resulting in an imbalance between vasodilatation and vasoconstriction [4]. Dysregulation of autonomic and sensitive nerve fibers, functional and structural vessel changes, intravascular alterations (platelet activation and impaired fibrinolysis, oxidative stress and reduced red blood cell plasticity, leukocyte hyper-activation) can be observed in the pathogenesis of RP [4].

\section{Neural abnormalities}

The autonomic nervous system regulates the vascular tone through a number of neurotransmitters, which trigger either vasodilation (calcitonin gene-related peptide, neurokinin A, substance $P$, and vasoactive intestinal peptide) or vasoconstriction (adrenergic agonists and nerve growth factor).

Calcitonin gene-related peptide (CGRP), an alternative product of the calcitonin gene, is a very potent vasodilator secreted by nerves that supply blood vessels. CGRP triggers the intracellular synthesis of adenylate cyclase in smooth muscle cells (SMC), which increases cyclic adenosine monophosphate (CAMP), ultimately leading to SMC relaxation. Decreased levels of the vasodilator CGRP in perivascular nerves of the finger skin have been demonstrated in PRP and SRP, and in vibration syndromes, suggesting its role in RP [9].

The vasodilator neurokinin A and substance $P$ can also be involved in the RP pathomechanism [10]. Neurokinin $A$ and substance $P$ induce an increase of CAMP, followed by SMC relaxation, and endothelial cyclic guanosine monophosphate (cGMP)-dependent nitric oxide accumulation, which strongly sustains the SMC relaxation already triggered by SMC-CAMP $[11,12]$.

The vasodilator vasoactive intestinal peptide (VIP), besides controlling a number of neurological functions, heart contractility and glycogenolysis, relaxes the SMC of the trachea, stomach, gallbladder and vessels. Terenghi et al. [13] observed a decreased VIP level in RP.

During local or whole body cooling, blood flow to the skin is reduced to prevent excessive heat loss. This effect is mediated by the reflex action of the sympathetic nervous system and appears to be abnormally augmented in RP. Based on pharmacological, biochemical and molecular approaches, three classes of $\alpha_{2}$-adrenoreceptors, $\alpha_{2 A}, \alpha_{2 B}$, and $\alpha_{2 C}$, have been described, each with a distinct distribution and function [14]. Chotani et al. [15] demonstrated that the $\alpha_{2 c}$ adrenoreceptor is the most important of the three in thermoregulation. It has been shown that the expression of these receptors on SMC of cutaneous arterioles is markedly increased following exposure to cold, inducing potent vasoconstriction, even in the presence of physiological levels of the $\alpha_{2 C}$ receptor agonist [16].

The role of the vasoconstrictor nerve growth factor (NGF) is intriguing. It shows elevated serum levels in SSc patients, but attempts to demonstrate a role in the generation of vasospasm in RP have not been successful [17-19].

\section{Functional and structural vessel changes}

In pRP, the vascular abnormalities are probably functional. By contrast, in SRP defective function and structural vascular abnormalities combine to compromise blood flow. Functional alterations of blood vessel tone can be mediated by endothelium-dependent and endothelium-independent factors. Among endothelium-dependent factors, decreased levels of the pleiotropic vasodilators nitric oxide (NO) and prostaglandin 12 (PGI2; prostacyclin) probably contribute to vasoconstriction in RP [20].

Nitric oxide, a pleiotropic mediator of inflammation, was discovered as a factor released from endothelial cells that caused vasodilatation by relaxing vascular smooth muscle and was therefore called endothelium-derived relaxing factor. Nitric oxide plays a critical role in the control of vascular tone generated constitutively by the endothelium as a messenger molecule which acts as an antithrombotic and cytoprotective agent, and also can cause tissue damage [21]. The impairment of constitutive $\mathrm{NO}$ synthesis and release as a consequence of vascular endothelium injury leads to 
dysregulation of vascular tone control and progressive disorganization of the vascular architecture $[6,20]$.

The role of NO in rheumatic diseases may be protective and anti-inflammatory or cytotoxic depending on the amount of $\mathrm{NO}$ produced and the targets available for action [22, 23].

Nitric oxide plays an important role in the vascular and cellular components of inflammatory responses. $\mathrm{Ni}$ tric oxide is a potent vasodilator by virtue of its action on vascular smooth muscle. Nitric oxide is an inhibitor of the migratory and proliferative activities of vascular smooth muscle cells. In addition, NO reduces platelet aggregation and adhesion, inhibits several features of mast cell-induced inflammation, and serves as an endogenous regulator of leukocyte recruitment. Blocking NO production under normal conditions promotes leukocyte recruitment. Thus, production of NO is an endogenous compensatory mechanism that reduces inflammatory responses. Abnormalities in endothelial production of $\mathrm{NO}$ occur in atherosclerosis, diabetes, and hypertension. Nitric oxide and its derivatives are microbiocidal, and thus NO is also a mediator of host defense against infection. Nitric oxide also causes tissue damage.

Small amounts of NO released by vascular endothelium regulate relaxation of adjacent smooth muscle and prevent leukocyte and platelet adhesion, but large amounts of $\mathrm{NO}$ released in response to cytokines can be cytotoxic $[22,23]$.

The endothelium also produces vasoconstrictors, such as endothelin-1 (ET-1), one of the most potent endogenous vasoconstrictors, which also causes vascular remodeling [24]. Release of ET-1 seems to be mediated by interleukin-6 (IL-6) and other pro-inflammatory cytokines [25]. Rajagopalan et al. [20] found significantly higher serum levels of ET-1 in patients with secondary Raynaud's disease. Moreover, overexpression of ET-1 has been reported in SSc-RP patients [26].

Angiotensin II is another vasoactive mediator (endothelial independent factor) which might contribute to RP. The vasoconstrictor angiotensin II (ATII) - an $\alpha$ adrenergic receptor agonist - can also promote structural vessel wall changes, including fibrosis [27]. Kawaguchi et al. [28] showed that serum ATII levels in patients with diffuse cutaneous SSc were significantly higher than in patients with limited cutaneous systemic sclerosis and healthy donors. ATII may promote tissue fibrosis by increasing synthesis of extracellular matrix components by fibroblasts [28].

Nailfold videocapillaroscopy (NVC) has been proposed as a first-line investigation in the early differential diagnosis between primary and secondary $\operatorname{RP}[29,30]$. Moreover, NVC is very helpful as a screening method for detecting patients with RP at high risk for the devel- opment of connective tissue disease [31, 32]. A critical, and possibly early event, often associated with a lympho-monocyte infiltration, is endothelial injury. Then, SMC abnormal migration in subendothelial regions and pericyte activation occur, contributing to vessel fibrosis and inducing abnormal synthesis of extracellular matrix components with intimal and perivascular fibrosis [33-35]. Endothelial proliferation, intimal thickening with progressive lumen obliteration and abnormal and ineffective neo-angiogenesis lead to vascular remodeling $[34,36,37]$. Similar vascular changes, with SMC hypertrophy and perivascular fibrosis but without intimal fibrosis, have been found in vibration syndrome RP [38].

\section{Angiogenesis}

Many studies have concentrated on the role of angiogenesis and microvascular endothelial injury in the pathogenesis of RP [4]. Among the angiogenesis inducers, vascular endothelial growth factor (VEGF) has been characterized as a key molecule. Vascular endothelial growth factor is involved in several steps of physiological and pathological angiogenesis including proliferation, survival and migration of endothelial cells [39].

Angiopoietin (Ang-1) is constitutively expressed in periendothelial cells (pericytes and smooth muscle cells). Under physiological conditions, Ang-1 has vasoprotective and anti-inflammatory actions, mediates vessel maturation and maintains vessel integrity by the recruitment of periendothelial cells [40].

Another important factor in endothelial activation is angiopoietin-2 (Ang-2), which causes vascular destabilization, thereby rendering the endothelium responsive to stimulation by inflammatory and angiogenic cytokines [41]. Angiopoietin-2 is stored and rapidly released by endothelial Weibel-Palade bodies upon stimulation by proinflammatory stimuli, hypoxia, shear stress and VEGF.

Angiopoietin-1 and Ang-2 have opposing effects. Ang-1 is required to maintain the endothelium in a quiescent state, whereas Ang-2 is a destabilizing factor considered to be pro-inflammatory. Angiopoietin-2 acts as a vessel-destabilizing cytokine, playing an essential role in vascular remodeling. Angiopoietin-2 and VEGF coordinately regulate endothelial behavior. In the presence of VEGF, Ang-2 enables migration and proliferation of endothelial cells and the sprouting of new blood vessels, but initiates endothelial cell death and vessel regression in the absence of proangiogenic activity [42]. Several studies have shown increased Ang-2 concentrations in vascular diseases, including SSc, compared to healthy volunteers [43]. Kuryliszyn-Moskal et al. [44] have suggested that Multiwave Locked System (MLS) laser therapy showed beneficial clinical effects and the tendency of 
normalization of Ang-2 concentration in the serum of RP patients. The authors suggested that Ang-2 serum level may be a useful marker of microvascular abnormalities in RP patients treated with MLS therapy [44].

\section{Intravascular disturbances}

Increased platelet aggregation and activation have been described in both systemic sclerosis and primary RP [45]. Molecules released by activated platelets include thromboxane A2, $\beta$-thromboglobulin, serotonin, platelet-derived microparticles, and platelet-derived growth factor. Augmented synthesis of platelet thromboxane A2, a potent vasoconstrictor and a platelet aggregator, has been reported in patients with RP, suggesting a possible mechanism [46]. Serotonin, another circulating vasoconstrictor released from platelets, has also been reported to be significantly elevated in patients with RP [47]. Fibrinolysis is normal in patients with primary RP, but in systemic sclerosis, reduced activity of the fibrinolytic system has been described [48, 49]. Impaired fibrinolysis documented by the increased serum levels of tissue plasminogen activator and elevated levels of von Willebrand factor are reported in secondary RP - mainly in patients with systemic sclerosis [45].

Oxidative stress has been implicated in the pathogenesis of RP. Repeated episodes of vasospasm followed by reperfusion are responsible for an intravascular accumulation of free radicals or reactive oxygen species (ROS) [50]. These substances mediate tissue damage and endothelial activation. Generated ROS might contribute to endothelium damage through peroxidation of cell membrane lipids in SSCRP, pRP and hand-arm vibration syndrome [50, 51].

The erythrocyte membrane is another substrate of intravascular ROS. Reduced red blood cell plasticity has been observed in SSc, but not in PRP [52].

Leukocyte activation has been reported in patients with SSc, pRP and hand-arm vibration syndrome and may contribute to oxidative stress [53].

\section{Nailfold videocapillaroscopy}

The first person to note the relationship between conjunctival inflammation and the presence of capillary disturbances, using a magnifying glass, was the Italian physician Giovanni Rasori [29]. Thanks to these descriptions, at the beginning of the $20^{\text {th }}$ century Hutchinson was able to differentiate RP into a primary phenomenon and secondary phenomenon by detecting microvascular changes [54].

In 1973, Maricq and LeRoy [55] published the first paper describing the specific capillaroscopic patterns in systemic sclerosis (SSc): the extent of microangiopathy detected by nailfold capillaroscopy has been shown to correlate with disease severity and prognosis. Abnormal capillary microscopy, the presence of certain autoantibodies such as anticentromere antibody, older age at onset of RP, severe vasospasm, and any signs of systemic disease are the predominant risk factors for the development of a connective tissue disease, predominantly SSc.

In the year 2000, Cutolo et al. [56] defined major nailfold videocapillaroscopy patterns that are useful when assessing the appearance and progression of sclerodermic microangiopathy.

Capillaroscopy is the most reliable way to distinguish between primary and secondary RP through identification of an early pattern of systemic sclerosis [57].

Morphological examination of skin capillaries is generally performed at the nailfold because this area is easily accessible for evaluation, and the major axis of the capillaries is parallel to the skin surface.

Nailfold videocapillaroscopy (NVC) is performed using a stereomicroscope. A fiber optic light source and filter provide cold illumination. The optical microscope may be connected to a color digital camera and a personal computer with a high-resolution color monitor (14 inches). Usually four consecutive fields extending over $1 \mathrm{~mm}$ in the middle of the nailfold are studied per finger using image analysis software. The images are taken at the time of examination and should be analyzed by the same experienced investigator without knowledge of the patient's clinical diagnosis.

Nailfold videocapillaroscopy of the fingers of both hands should be performed following a period of acclimatization lasting at least 20 minutes at a room temperature of $20-24^{\circ} \mathrm{C}$. To obtain the best visibility of the microvasculature a drop of immersion oil is recommended. Only the capillaries in the distal row of the nailfold are analyzed.

The usual capillaroscopic features are 10-30 capillaries/1 mm, with typical hairpin-shaped loops arranged in parallel rows. Isolated tortuous, meandering and elongated capillaries can be found in healthy controls also.

The evaluation of morphological changes includes such parameters as: loop density, capillary length variability, percentage of loops with architectural derangement, i.e. tortuous, meandering, enlarged/giant, ramified or bushy capillaries, irregular distribution of the capillary array and the presence of extravasations into perivascular tissue. In recent studies an attempt of the classifications [30-32] of morphological changes has been proposed: dilated capillaries - microvessels with a diameter of the arterial limb wider than $0.015 \mathrm{~mm}$ $(=15 \mu \mathrm{m})$ or a venous limb wider than $0.020 \mathrm{~mm}$ (= $20 \mu \mathrm{m})$, giant capillary loops - homogeneously enlarged loops with a diameter wider than $0.050 \mathrm{~mm}$ $(=50 \mu \mathrm{m})$, elongated capillaries - microvessels with 
length greater than $0.500 \mathrm{~mm}(=500 \mu \mathrm{m})$, microhemorrhage - presence of a dark red mass characterized by hemosiderin deposit derived from capillary injury, tortuosity - a variation of the typical hairpin capillary shape and avascular area - a distance between two adjacent capillary loops longer than $0.500 \mathrm{~mm}$ (= $500 \mu \mathrm{m})$.

In our examinations the capillaroscopic findings are graded from 0 to 3, where: score 0 indicates normal features (> 8 capillaries/mm, typical hairpin-shaped loops arranged in parallel rows), 1 indicates mild (6-8 capillaries $/ \mathrm{mm}$, < 33\% changed capillary loops with nonhomogeneous distribution or size of loops, elongation of the loop or shortened loops, and the absence of hemorrhages), 2 indicates moderate (33-66\% morphologically changed capillaries, enlarged loops, diminished loop density and microhaemorrhages), and 3 indicates severe changes (more than $66 \%$ dilated capillaries with heterogeneous features of angiogenesis with a variable capillaroscopic pattern, avascular areas and microhemorrhages). The mean score for each subject is obtained from the analysis of all ten fingers [58, 59].

Nailfold capillaroscopy has enormous potential in the assessment of digital vascular disease. Widefield microscopy is inexpensive, easy to use, and useful prognostically in patients presenting with RP [60]. Direct visualization of capillaries of different patient subgroups may raise fundamental questions regarding pathogenesis. Videocapillaroscopy is an extension of the widefield technique which allows quantitation of these abnormalities: using a video camera and digitizing system, dimensions of individual capillaries can be measured, thus offering the potential of a non-invasive technique which should allow objective assessment of microvascular disease and its progression. The technique of videocapillaroscopy facilitates the quantitation of the nailfold capillary abnormalities which have been long recognized in patients with connective tissue disease. Because images are recorded on video, images can be stored for analysis and reanalysis.

Videocapillaroscopy may allow quantitation of microvascular disease progression over time and therefore be an important research tool. This technique is useful for assessing early diagnosis and providing prognostic information, and is capable of differentiating between active and inactive forms of RP, and also of monitoring disease progression.

The authors declare no conflict of interest.

\section{References}

1. Pope JE. The diagnosis and treatment of Raynaud's phenomenon: a practical approach. Drugs 2007; 67: 517-525.
2. Edwards JM, Taylor LM, Porter JM, et al. Raynaud's syndrome and upper extremities small artery occlusive diseases. In: Vascular surgery: principle and practice. Hobson RW, Wilson SE, Veith FJ (eds.). Marcell Dekker, New York 2004; 903-912.

3. Katada Y, Tanaka T. Images in clinical medicine. Lingual Raynaud's phenomenon. N Engl J Med 2012; 366: e12.

4. Herrick AL. Pathogenesis of Raynaud's phenomenon. Rheumatology 2005; 44: 587-596.

5. Amanzi L, Braschi F, Fiori G, et al. Digital ulcers in scleroderma: staging, characteristics and sub-setting through observation of 1614 digital lesions. Rheumatology 2010; 49: 1374-1382.

6. Block JA, Sequeira W. Raynaud's phenomenon. Lancet 2001; 357: 2042-2048.

7. Lim MJ, Kwon SR, Kim SC, et al. A case of MCTD overlapped by Takayasu's arteritis, presenting Raynaud's phenomenon as the initial manifestation of both diseases. Rheumatol Int 2009; 29: 685-688.

8. Goundry B, Bell I, Langtree A, et al. Diagnosis and management of Raynaud's phenomenon. BMJ 2012; 344: e289.

9. Bunker CB, Goldsmith PC, Leslie TA, et al. Calcitonin gene-related peptide, endothelin-1, the cutaneous microvasculature and Raynaud's phenomenon. Br J Dermatol 1996; 134: 399 406.

10. Matucci-Cerinic M, Pietrini U, Marabini S. Local vasomotor response to intravenous infusion of substance $P$ and glyceryl trinitrate in systemic sclerosis. Clin Exp Rheumatol 1990; 8: 561-565.

11. Quartara L, Maggi CA. The tachykinin NK1 receptor. Part I: ligands and mechanisms of cellular activation. Neuropeptides 1997; 31: 537-563.

12. Quartara L, Maggi CA. The tachykinin NK1 receptor. Part II: distribution and pathophysiological roles. Neuropeptides 1998; 32: 1-49.

13. Terenghi G, Bunker CB, Liu YF, et al. Image analysis quantification of peptide-immunoreactive nerves in the skin of patients with Raynaud's phenomenon and systemic sclerosis. J Pathol 1991; 164: 245-252.

14. Flavahan NA, Flavahan S, Mitra S, et al. The vasculopathy of Raynaud's phenomenon and scleroderma. Rheum Dis Clin North Am 2003; 29: 275-291.

15. Chotani MA, Flavahan S, Mitra S, et al. Silent alpha(2C)-adrenergic receptors enable cold-induced vasoconstriction in cutaneous arteries. Am J Physiol Heart Circ Physiol 2000; 278: H1075-H1083.

16. Flavahan NA, Flavahan S, Liu Q, et al. Increased alpha-2-adrenergic constriction of isolated arterioles in diffuse scleroderma. Arthritis Rheum 2000; 43: 1886-1896.

17. Matucci-Cerinic M, Giacomelli R, Pignone A, et al. Nerve growth factor and neuropeptides circulating levels in systemic sclerosis (scleroderma). Ann Rheum Dis 2001; 60: 487-494.

18. Lise MC, Sparsa A, Marie I, et al. Serum neurotropin profile in systemic sclerosis. PLoS One 2010; 5: e13918.

19. Klein-Weigel P, Gutsche-Petrak B, Humpel C, et al. Circulating nerve growth factor in primary and secondary Raynaud's syndrome - results of a pilot study. Vasa 2009; 38: 39-45.

20. Rajagopalan S, Pfenninger D, Kehrer C, et al. Increased asymmetric dimethylarginine and endothelium 1 levels in secondary Raynaud's phenomenon: Implications for vascular dys- 
function and progression of disease. Arthritis Rheum 2003; 48: 1992-2000.

21. Takagi K, Kawaguchi Y, Hara M, et al. Serum nitric oxide (NO) levels in systemic sclerosis patients: correlation between NO levels and clinical features. Clin Exp Immunol 2003; 134: 538-544.

22. Clancy RM, Amin AR, Abramson SB. The role of nitric oxide in inflammation and immunity. Arthritis Rheum 1998; 41: 11411151.

23. Matucci Cerinic M, Kahaleh MB. Beauty and the best. The nitric oxide paradox in systemic sclerosis. Rheumatology 2002 41: 843-847.

24. Kirchengast M, Munter K. Endothelin-1 and endothelin receptor antagonists in cardiovascular remodeling. Proc Soc Exp Biol Med 1999; 221: 312-325.

25. Rychlik-Golema W, Mastej K, Adamiec R. The role of endothelin-1 and selected cytokines in the pathogenesis of Raynaud's phenomenon associated with systemic connective tissue diseases. Int Angiol 2006; 25: 221-227.

26. Vancheeswaran R, Azam A, Black C, et al. Localization of endothelin-1 and its binding sites in scleroderma skin. J Rheumato 1994; 21: 1268-1276.

27. Duprez DA, Role of the renin-angiotensin-aldosterone system in vascular remodeling and inflammation: a clinical review. J Hypertens 2006; 24: 983-991.

28. Kawaguchi Y, Takagi K, Hara M, et al. Angiotensin II in the lesional skin of systemic sclerosis patients controbutes to tissue fibrosis via angiotensin II type 1 receptors. Arthritis Rheum 2004; 50: 216-226.

29. Cutolo M, Grassi W, Matucci Cernic M. Raynaud's phenomenon and the role of capillaroscopy. Arthritis Rheum 2003; 48 : 3023-3030.

30. Lambova SN, Müller-Ladner U. The role of capillaroscopy in differentiation of primary and secondary Raynaud's phenomenon in rheumatic diseases: a review of the literature and two case reports. Rheumatol Int 2009; 29: 1263-1271.

31. Meli M, Gitzelmann G, Koppensteiner R, et al. Predictive value of nailfold capillaroscopy in patients with Raynaud's phenomenon. Clin Rheumatol 2006; 25: 153-158.

32. Kuryliszyn-Moskal A, Klimiuk PA, Sierakowski S, et al. Vascular endothelial growth factor (VEGF) in systemic lupus erythematosus: relationship to disease activity, systemic organ manifestation, and nailfold capillaroscopic abnormalities. Arch Immunol Ther Exp 2007; 55: 179-185.

33. Rajkumar VS, Sundberg C, Abraham DJ, et al. Activation of microvascular pericytes in autoimmune Raynaud's phenomenon and systemic sclerosis. Arthritis Rheum 1999; 42: 930-941.

34. Cipriani P, Marrelli A, Benedetto PD, et al. Scleroderma Mesenchymal Stem Cells display a different phenotype from healthy controls; implications for regenerative medicine. Angiogenesis 2013; 16: 595-607.

35. Sunderkotter C, Riemekasten G. Pathophysiology and clinical consequences of Raynaud's phenomenon related to systemic sclerosis. Rheumatology 2006; 45 (Suppl.3): iii33-iii35.

36. Liakouli V, Cipriani P, Marelli A, et al. Angiogenic cytokines and growth factors in systemic sclerosis. Autoimmun Rev 2011; 10: 590-594.

37. Blaise $S$, Maas R, Trocme C, et al. Correlation of biomarkers of endothelium dysfunction and matrix remodeling in patients with systemic sclerosis. J Rheumatol 2009; 36: 984-988.
38. Takeuchi T, Futatsuka M, Imanishi H, et al. Pathological changes observed in the finger biopsy of patients with vibration-induced white finger. Scand J Work Environ Health 1986; 12: 280-283.

39. Paleolog EM. The vasculature in rheumatoid arthritis: cause or consequence? Int J Exp Pathol 2009; 90: 249-261.

40. Yancopoulos GD, Davis S, Gale NW, et al. Vascular-specific growth factors and blood vessel formation. Nature 2000; 407: 242-248.

41. Fiedler U, Augustin HG. Angiopoietins: a link between angiogenesis and inflammation. Trends Immunol 2006; 27: 552558.

42. Kurosaka D, Hirai K, Nishioka M, et al. Clinical significance of serum levels of vascular endothelial growth factor, angiopoietin-1, and angiopoietin- 2 in patients with rheumatoid arthritis. J Rheumatol 2010; 37: 1121-1128.

43. Michalska-Jakubus M, Kowal-Bielecka O, Chodorowska G, et al. Angiopoietin-1 and -2 are differentially expressed in the sera of patients with systemic sclerosis: high angiopoietin-2 levels are associated with greater severity and higher activity of the disease. Rheumatology 2011; 50: 746-755.

44. Kuryliszyn-Moskal A, Kita J, Dakowicz A, et al. The influence of Multiwave Locked System (MLS) laser therapy on clinical features, microcirculatory abnormalities and selected modulators of angiogenesis in patients with Raynaud's phenomenon. Clin Rheumatol 2015; 34: 489-96.

45. Silveri F, De Angelis R, Poggi A, et al. Relative roles of endothelial cell damage and platelet activation in primary Raynaud's phenomenon (RP) and RP secondary to systemic sclerosis. Scand J Rheumatol 2001; 30: 290-296.

46. Hutton RA, Mikhailidis DP, Berenstein RM, et al. Assessment of platelet function in patients with Raynaud's syndrome. J Clin Pathol 1984; 37: 182-187.

47. Biondi ML, Marasini B, Bianchi E, et al. Plasma free and intraplatelet serotonin in patients with Raynaud's phenomenon. Int J Cardiol 1988; 19: 335-339.

48. Herrick AL, Illingworth K, Blann A, et al. Von Willebrand factor, thrombomodulin, thromboxane, beta-thromboglobulin and markers of fibrinolysis in primary Raynaud's penomenon and systemic sclerosis. Ann Rheum Dis 1996; 55: 122-127.

49. Lau CS, McLaren M, Mackay I, et al. Baseline plasma fibrinolysis and its correlation with clinical manifestations in patients with Raynaud'sphenomenon. Ann Rheum Dis 1993; 52: 443-448.

50. Herrick AL, Matucci CM. The emerging problem of oxidative stress and the role of antioxidants in systemic sclerosis. Clin Exp Rheumatol 2001; 19: 4-8.

51. Stoyneva Z, Lyapina M, Tzvetkov D, et al. Current pathophysiological views on vibration-induced Raynaud's phenomenon. Cadiovasc Res 2003; 57: 615-624.

52. Solans R, Motta C, Sola R, et al. Abnormalities of erythrocyte membrane fluidity, lipid composition, and lipid peroxidation in systemic sclerosis: evidence of free radical-mediated injury. Arthritis Rheum 2000; 43: 894-900.

53. Lau CS, O'Dowd A, Belch JJ. White blood cell activation in Raynaud's phenomenon of systemic sclerosis and vibration white finger. Ann Rheum Dis 1992; 51: 249-252.

54. Hutchinson H. Raynaud's phenomenon. La Presse medicale 1901; 71: 403-405. 
55. Maricq HR, LeRoy EC. Patterns of finger capillary abnormalities in connective tissue disease by 'widefield' microscopy. Arthrits Rheum 1973; 16: 619-628.

56. Cutolo M, Sulli A, Pizzorni C, et al. Nailfold videocapillaroscopy assessment of microvascular damage in systemic sclerosis. J Rheumatol 2000; 27: 155-160.

57. De Angelis R, Grassi W, Cutolo M. A growing need for capillaroscopy in Rheumatology. Arthritis Rheum (Arthritis Care Res) 2009; 61: 405-410.

58. Ingegnoli F, Boracchi P, Gualtierotti R, et al. Improving outcome prediction of systemic sclerosis from isolated Raynaud's phenomenon: role of autoantibodies and nail-fold capillaroscopy. Rheumatology 2010; 49: 797-805.

59. Herrick AL, Cutolo M. Clinical implications from capillaroscopic analysis in patients with Raynaud's phenomenon and systemic sclerosis. Arthritis Rheum 2010; 62: 2595-2604.

60. Górska A, Bartnicka M, Rutkowska-Sak L. Value of nailfold videocapillaroscopy and high-sensitivity Creactive protein test in diagnosis of microangiopathy in adoscents with Raynaud's phenomenon. Reumatologia 2013; 51: 189-194. 